\title{
Motor Commands of Facial Expressions: The Bereitschaftspotential of Posed Smiles
}

\author{
Sebastian Korb · Didier Grandjean · \\ Klaus Scherer
}

Accepted: 11 February 2008/Published online: 12 March 2008

(C) Springer Science+Business Media, LLC 2008

\begin{abstract}
Electroencephalographic (EEG) premotor potentials with negative polarity like the Bereitschaftspotential (BP) are known to precede self-paced voluntary movements of the limbs and other body parts. This is however the first report of such premotor potentials before posed smiles. Scalp EEG was recorded in 16 healthy participants performing self-paced unilateral and bilateral smiles and unilateral finger movements. Amplitudes over six central electrodes and voltage distributions over the entire scalp were compared across conditions at time of EMG-onset, thus focusing on the late BP. Results show the presence of a premotor potential before posed smiles with a later onset, symmetrical bilateral distribution, and smaller amplitude at time of movement-onset, compared to finger movements. Future studies should investigate the BP before various types of emotional and non-emotional facial expressions.
\end{abstract}

Keywords Movement preparation .

Bereitschaftspotential - Facial expression - EEG ·

Smile $\cdot$ Facial EMG

\section{Introduction}

Facial expression is one of the components of emotional episodes [1], and of great interest to psychologists and neuroscientists as it may reflect a person's inner feelings. However, facial expressions are not always a window to

S. Korb $(\square) \cdot$ D. Grandjean · K. Scherer Department of Psychology, Swiss Center for Affective Sciences, University of Geneva, 7, rue des Battoirs, Geneva 1205, Switzerland

e-mail: Sebastian.Korb@pse.unige.ch the soul, as most people are-at various levels of proficiency-able to (1) display an emotional facial expression without feeling the corresponding emotion, or (2) modulate (e.g. suppress) an upcoming emotional facial expression triggered by an emotional episode [2]. The study and differentiation of these various types of "emotional" facial expression has mainly been carried out at the peripheral level by comparing patterns of muscular contractions in the face. However, a double dissociation between volitional and emotional facial paresis suggests that voluntary and spontaneous facial expressions rely on partly distinct neural circuitries [3, 4]. One way to investigate the neural differences between different types of facial expressions may be to focus onto motor and premotor activities in the CNS, and more specifically onto the Bereitschaftspotential (BP). In the here reported study, our aim was to assess whether voluntary posed smiles are preceded by a BP, which we compared to the premotor potentials of right and left index-finger movements, as they are frequently used in BP-paradigms. Moreover, we included conditions with right and left unilateral smiling movements in order to assess the differences between unilateral and bilateral facial movements.

The Bereitschaftspotential (BP) is an electroencephalographic potential with negative polarity that precedes movements, and has mainly been studied in relation to voluntary, self-paced limb movements [5]. However, recent studies have found a BP also before imagined, not executed movements [6-8], and others suggest that spontaneous movements may be preceded by a BP as well $[9,10]$. The BP can be further subdivided [11] into an 'early BP', characterized by a slowly rising negative curve, followed by a 'late BP' consisting of a steeper Negative Slope (NS') peaking into the Motor Potential (MP) at about time of EMG-onset. Early BP is symmetrically and widely 
distributed over the scalp, and reaches maximal amplitude over the midline centro-parietal area (e.g. see [12]). Its probable sources are in the cortical medial-wall motor areas. For hand movements, the late BP becomes maximal over contralateral central areas and originates mainly in the primary motor cortex (M1).

Previous studies suggest that BP at the scalp reaches greater amplitude around time of movement onset for bilateral than for unilateral finger and hand movements, and furthermore remains symmetrically distributed over both hemispheres for bilateral movements. For example, Cui \& Deecke [13] analyzed brain topography using current source density mapping in healthy subjects. For bilateral index-finger extensions, the authors reported symmetrical and bilateral voltage distributions of all premotor components, most of which were also of higher amplitude, then for unilateral movements. Ikeda et al. [14] studied three patients with subdural electrodes over the SMA and the motor cortex, and reported a larger MP for bilateral than for unilateral hand and foot movements. At least two distinct factors may lead to higher premotor potential amplitudes for bilateral than unilateral movements: (1) the amount of muscle mass activated for performing the movement, and (2) the increased difficulty of performing coordinated bilateral movements. However, the factor task-difficulty seems to be-at least under certain circumstances-more relevant. For example, Kitamura, Shibasaki, and Kondo [15] reported significantly larger amplitudes of NS' at the contralateral precentral area of the scalp for isolated middle-finger movements, than for simultaneous middle- and indexfinger movements. Importantly, participants in this experiment reported the two-finger movement (which yet required the activation of a greater muscle mass and probably also of a greater part of the hand somatosensory area) to be less difficult to execute than isolated middlefinger movements.

A BP has also been recorded before voluntary self-paced horizontal eye saccades [16], swallowing movements [17], jaw movements [18, 19], and lip movements [20]. Huckabee and colleagues [11], for example, found for voluntary swallowing movements a BP with symmetrical distribution, absence of the NS', and smaller amplitude than for unilateral finger movements. Yamamoto et al. [16] showed the BP preceding eye saccades to be of lower amplitude than the one before unilateral hand movements. However, smaller BP amplitudes (compared to unilateral limb movements) of both saccades and swallowing may be due to the fact that these movements are not solely performed via pyramidal tracts, but consist partly of reflexive behavior. Yoshida and colleagues [19] found larger BPs before right and left lateral mandibular movements than before mouth opening and closing. However, mandibular movements are mainly controlled by the trigeminal and not by the facial nerve, a finger movement condition was not included, and the amplitude differences may be attributable to the greater difficulty of lateral mandibular movements. In summary, although previous studies described BPs before movements in the area of the face or head (and partly compared them to finger and limb movements) it remains unclear whether bilateral posed smiles should result in greater or smaller amplitudes than unilateral finger movements.

In the present study we investigated the presence of a BP before posed smiles, unilateral smiling movements and unilateral index-finger movements. We used unilateral smiles for comparison with the well-known paradigm of unilateral finger movements. Specifically, we focused on topographies and amplitudes at time of EMG onset, thus targeting NS' and MP (the late BP). As these components are usually stronger over the contralateral hemisphere for unilateral finger movements [11], (1) we expected to find greater amplitudes over $\mathrm{C} 1$ for right-sided and over the $\mathrm{C} 2$ electrode for left-sided finger movements. In analogy to unilateral finger movements, and based on the fact that the neuromotor representations in M1 are more laterally located for the (lower) face than for the fingers [4, 21], (2) we expected amplitudes over C3 to be stronger compared to $\mathrm{C} 4$ for right-sided, and weaker for left-sided smiles. On the basis of previous studies comparing eye-saccades and swallowing movements to unilateral hand or finger movements [16, 17], and others comparing unilateral to bilateral limb movements [13], (3) we expected bilateral smiles to generate a negative potential of more symmetrical, bilateral distribution compared to the one preceding unilateral finger and smiling movements. Moreover, because the amplitude of the late BP is related to the complexity of the motor task [11], and unilateral smiles were reported by our participants to be more difficult to execute than bilateral smiles, (4) we predicted negativity at $\mathrm{Cz}$ to be significantly lower for bilateral than for unilateral smiles. Lastly, (5) we expected to find a specific topographical pattern of voltage distributions for each condition.

\section{Methods}

\section{Participants}

Twenty-one right-handed voluntary participants (4 males, mean age $=22.5 \pm 3.2$ ) were recruited in the graduate and post-graduate population of the University of Geneva and gave informed consent for participation in the study. They were all free of a history of neurological or psychiatric disorders. Four participants were excluded because no clear BP preceding left and/or right finger movements was 
discernable in their averaged potentials. A fifth one was excluded due to technical failure. Data from 16 participants was included in the final analyses.

\section{Procedure}

Participants were seated in a relaxed position, facing a blank wall (approx. $3 \mathrm{~m}$ distant), with their arms resting on armrests and their hands comfortably positioned on their legs. They performed five experimental blocks, lasting $5 \mathrm{~min}$ each, and implying the repetitive performance of brisk, short movements, starting from a resting state, and being followed by approximately $5 \mathrm{~s}$ of rest. All movements were self-paced and not triggered by any external cue. Therefore, the precise timing of and intervals between movements could vary slightly between participants and blocks. The average number of trials per block was 51.2 (range 39-66). Movements per block were: right index finger elevation (finR), left index finger elevation (finL), right-sided smile (zygR), left-sided smile (zygL), and bilateral smile (smile). Instructions were given to reduce blinking and eye movements to a minimum, and not to evoke any amusing or other emotional thoughts. Order of blocks was counterbalanced across participants.

\section{EEG and EMG Recordings and Analyses}

We recorded the EEG from 64 scalp channels using an ActiveTwo BioSemi system, with $\mathrm{Ag} / \mathrm{AgCl}$ active electrodes, $2048 \mathrm{~Hz}$ sampling rate, and an electrical reference from the left and right mastoids. Vertical electrooculogram (VEOG) was taken from above and beneath the left or right eye. Bipolar surface EMG (interelectrode distance $1 \mathrm{~cm}$ ) was recorded bilaterally from the extensor digitorum muscle over the forearm and from the zygomaticus major muscle over the cheek. Data preprocessing and analysis was done using Vision Analyzer software (Brain Vision, Munich, Germany) and Cartool software by Denis Brunet (http://brainmapping.unige.ch/Cartool.htm), which allows the segmentation (based upon statistical parameters) of ERPs into periods (functional micro-states) of relative stability (see [22] for a review, and [23] for a recent example).

Offline, EMG data were digitally bandpass-filtered from $20 \mathrm{~Hz}$ to $400 \mathrm{~Hz}$ [24] and rectified. Onset of EMG was automatically marked in each EMG-channel based on an amplitude threshold of $30 \mu \mathrm{V}$, and verified through visual inspection. For each block, trials with activity in a recorded but unrelated muscle were excluded.

EEG and VEOG data were re-referenced to digitally linked mastoids, filtered from $0.1 \mathrm{~Hz}$ to $30 \mathrm{~Hz}$, and segmented from $2500 \mathrm{~ms}$ before to $500 \mathrm{~ms}$ after EMG onset.
Noisy electrodes (in average 1.7 per condition and participant) were interpolated in Cartool. Importantly, our electrodes of interest (see beneath) did not require interpolation. Semi-automatic ocular correction was done with the Gratton-Coles algorithm [25] and checked by visual inspection. Following baseline correction (from 2500 to $2000 \mathrm{~ms}$ before EMG onset), all epochs were checked visually, and those containing important artifacts $(> \pm 120 \mu \mathrm{V})$ before EMG-onset were excluded. Artifacts occurring after EMG-onset, as they could occur during facial movements, were not taken into consideration. Mean and standard deviation of rejected trials per condition were: finL(8.3; 8.8), finR(6.3; 6.2), smile(8.8; 6.8), $\operatorname{zygL}(6.1$; 6.8), zygR(6.1; 5.1). A similar $\left(\mathrm{X}^{2}(4)=.31, P=\right.$ n.s. $)$ number of trials was included into analyses for each condition: finL $(M=41.8 ; S D=10)$, finR $(M=41.8 ; S D=$ 11.5), smile( $\mathrm{M}=46.4 ; \mathrm{SD}=8)$, $\operatorname{zygL}(44.6 ; \mathrm{SD}=8.5)$, $\operatorname{zygR}(\mathrm{M}=45.8 ; \mathrm{SD}=6.3)$. Amplitudes at time of EMG onset were extracted at electrodes $\mathrm{C} 1, \mathrm{C} 2, \mathrm{C} 3, \mathrm{C} 4, \mathrm{Cz}$, and $\mathrm{FCz}$ for the average potential of each participant and condition. Greenhouse-Geisser corrections were applied in the case of sphericity violations.

\section{Results}

Amplitudes

A repeated measures ANOVA carried out on the ERPamplitudes at time of EMG onset with the factors Condition (5 levels) and Electrodes (6 levels) revealed a main effect of Condition $(\mathrm{F}(4,60)=4.16, P<.05)$, a main effect of Electrode $(\mathrm{F}(5,75)=7.57, P<.001)$, and more interestingly an Electrode by Condition interaction $(\mathrm{F}(20,300)=6.11, P<.001)$. Post hoc tests (contrasting all 5 conditions) with Bonferroni correction revealed that the zygL condition produced greater negativity than the finR $(P=.01)$ and the smile condition (all $P<.05)$, over all electrodes. Moreover, independently of condition, negativity over $\mathrm{Cz}$ (mean $=-6.78$ ) was greater than over C3 (-4.87) and C4 $(-5.20)$ (all $P<.001)$, and the signal over C3 was weaker than over C1 $(-6.17)$ and C2 $(-6.01)$ (all $P<.05$ ).

As expected, we found a shift to the contralateral hemisphere for unilateral finger movements (see Fig 1), as the planned contrast finL $(\mathrm{C} 1>\mathrm{C} 2)>\operatorname{finR}(\mathrm{C} 1>\mathrm{C} 2)$ confirmed negativity over $\mathrm{C} 1(-5.40)$ during right-finger movements to be greater than over $\mathrm{C} 2(-2.85)$, while the opposite, i.e. greater negativity over $\mathrm{C} 2(-8.24)$ than $\mathrm{C} 1$ $(-6.14)$, was true for left-sided finger movements $(\mathrm{F}(1,15)=30.87, P<.001)$. A similar shift to the contralateral hemisphere had been expected for unilateral smiles. To test this hypothesis we contrasted amplitudes at 
electrodes $\mathrm{C} 3$ and $\mathrm{C} 4$, in light of the fact that the neural representations of facial muscles are situated more laterally in the primary motor cortex compared to the representations of the fingers [4]. However, the contrast
zygL $(\mathrm{C} 3>\mathrm{C} 4)>\operatorname{zygR}(\mathrm{C} 3>\mathrm{C} 4)$ was only marginally significant $(\mathrm{F}(1,15)=3.52, P=.08)$.

In order to test our hypothesis that bilateral smiling movements elicit a more symmetrical, less contralateral
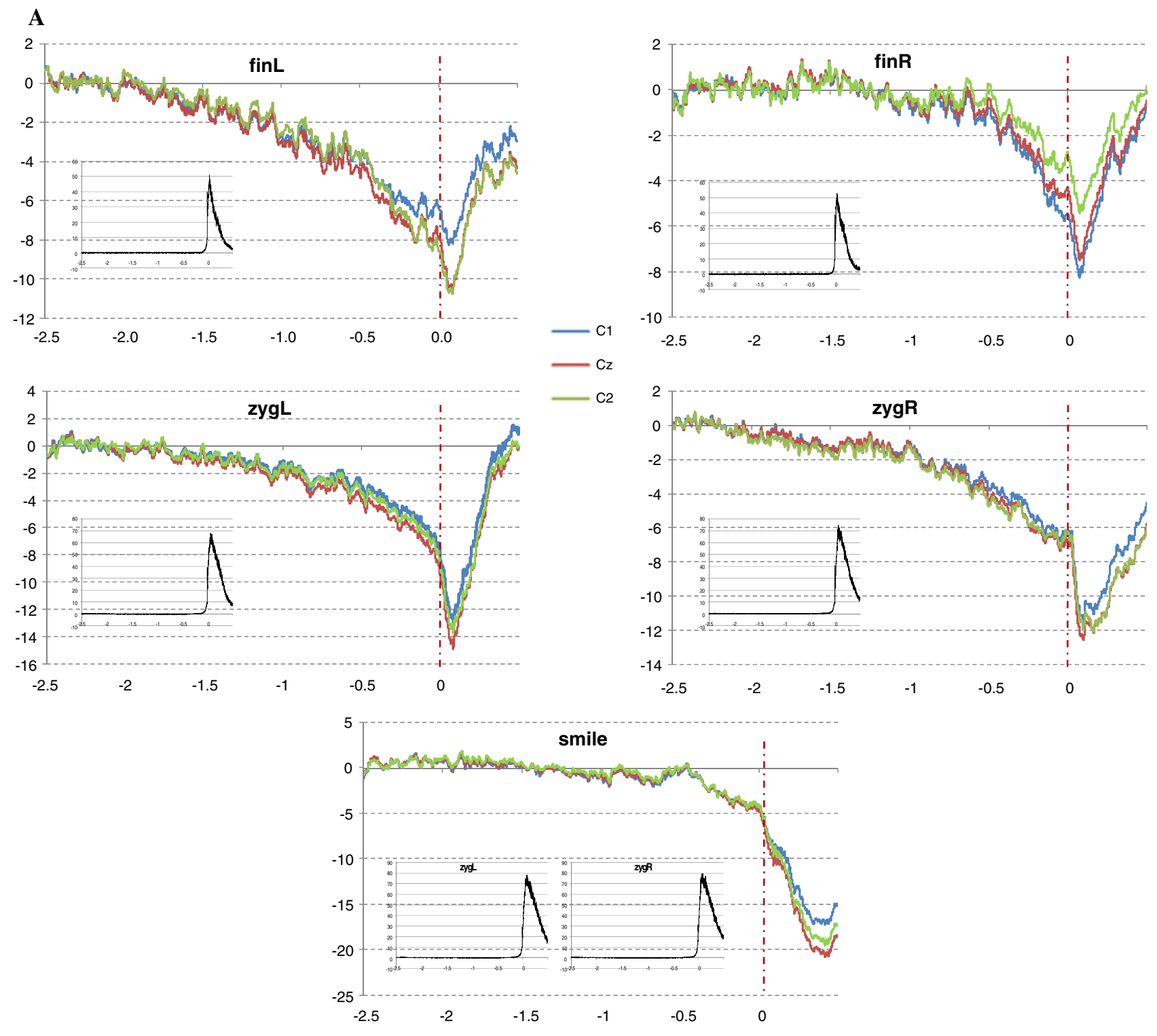

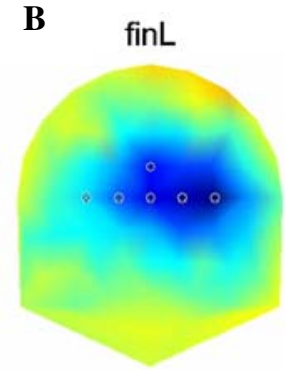

Fig. 1 (a) Average $\mathrm{BP}$ (in $\mu \mathrm{V}$ ) at electrode sites $\mathrm{C} 1$ (blue), $\mathrm{Cz}$ (red), and C2 (green). EMG onset marked by red dashed bar (time 0 ). Average EMG (over same time window as EEG) of corresponding muscle(s) superimposed. (b) Average potential maps seen
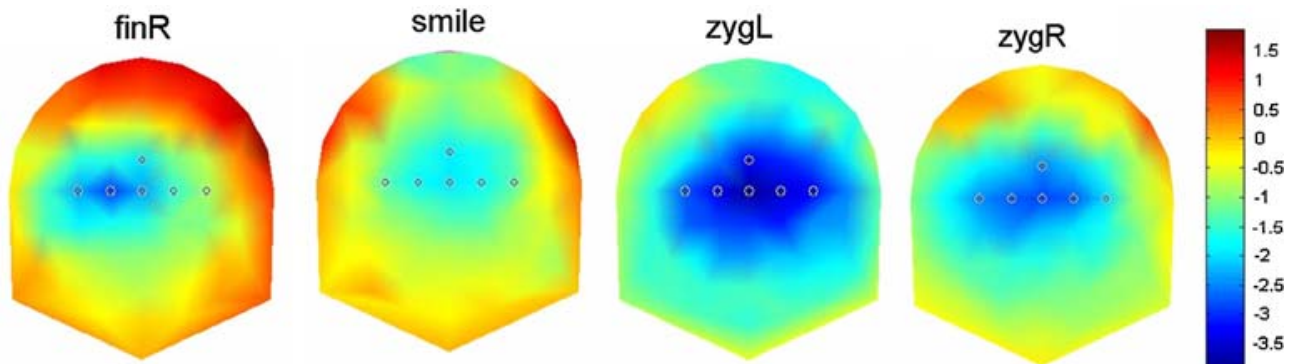

from the top. Nose is up, negativity in blue. Circles show electrodes of interest. Note the marked negativity shift to the contralateral hemisphere for finger movements, and slightly less for unilateral smiling. Topography of bilateral smile is symmetrical 
late $\mathrm{BP}$ we subtracted, for each condition and at time of EMG-onset, amplitudes at electrode $\mathrm{C} 4$ from amplitudes at electrode $\mathrm{C} 3$, and compared them to 0 (the score expected for an absence of lateral shift to either hemisphere) via t-tests (one-tailed, except for the condition smile). The laterality coefficients of the conditions finL, finR, and zygR were significantly different from 0 (all $P<.05$ ), revealing that the late BP was stronger over the contralateral hemisphere, but the conditions zygL and bilateral smile were not, suggesting that these conditions produced a more symmetrical BP.

As expected, unilateral smiles, which participants reported to be more difficult to execute, elicited greater negativity over $\mathrm{Cz}(-10.14$ and -7.09 for $z y g L$ and $z y g R)$ than trials requiring bilateral smiles $(-4.64)(\mathrm{F}(1,15)=$ $5.38, P<.05)$. The same contrast was marginally significant at the more rostral electrode site $\mathrm{FCz}(\mathrm{F}(1,15)=3.68$, $P=.07)$. Moreover, post-hoc tests with Bonferroni correction revealed significantly higher signal amplitude for left $(-7.73)$ than for right finger movements $(-4.33)$ at electrode $\mathrm{Cz}(P<.01)$. Amplitudes for bilateral smiling $(-4.64)$ and right finger movements at electrode $\mathrm{Cz}$ did not differ from each other $(P=$ n.s. $)$.

\section{Topographies}

On the grand averages topographic maps were extracted at time of EMG onset (averaging a 20 ms-window: $10 \mathrm{~ms}$ before to $10 \mathrm{~ms}$ after the EMG onset). These 5 maps were fitted back (using Cartool software) onto the average potentials of each participant on the same $40 \mathrm{~ms}$ time window. A repeated measures ANOVA with the factors Condition (5 levels) and Map (5 levels) was carried out on the Global Explained Variance (GEV) of the fitting procedure, resulting in a main effect of Map $(\mathrm{F}(4,60)=2.68, \quad P<.05)$, and a Condition by Map interaction $(\mathrm{F}(16,240)=8.56, P<.001, \varepsilon=.34)$. Planned comparisons were carried out to test the specificity of each map for its corresponding condition [22], e.g. finL(Map finL) $>$ finL(all other maps). Results showed that, with exception of the voltage map for right-sided smiles $(\mathrm{F}(1,15)=1.36, P=$ n.s. $)$, each map attained the highest degree of GEV for its corresponding condition, compared to all other maps, all $\mathrm{F}(1,15)>6.44$, all $P<.05$.

A similar ANOVA on the Global Field Power (GFP) did not result in any significant main effects, but revealed a significant Condition by Map interaction $(\mathrm{F}(16,240)=$ 7.14, $P<.001, \varepsilon=.46)$. With exception of the map for right-sided smiles $(\mathrm{F}(1,15)<1)$, each voltage map led to the greatest GFP for its corresponding condition (same planned comparisons method as for GEV), compared to all other maps, all $\mathrm{F}(1,15)>7.9$, all $P<.05$.

\section{Discussion}

The purpose of this study was to investigate whether a BP, which can be seen before finger, hand, or foot movements, is also present befor posed smiles. We used unilateral smiling and index-finger movements for comparison.

The results mirror previous findings concerning the neural correlates of motor preparation of unilateral, voluntary, self-paced finger movements, both in terms of single traces [26], and scalp topographies [27]. Indeed, we showed a classical BP starting approximately $1.4 \mathrm{~s}$ before EMG-onset for the right finger, and two seconds before EMG for the left finger. Amplitudes of this slow negative potential showed, at time of EMG-onset, a shift to the contralateral hemisphere. On average, greatest amplitude was found at the vertex $(\mathrm{Cz}$, but only difference with electrodes C3 and C4 was significant in post-hoc tests). This finding, which we had not anticipated, has already been reported by others [13].

To the best of our knowledge, this is the first report of a BP preceding posed smiles and unilateral smiling movements. Left-sided and right-sided unilateral smiling movements produced higher amplitudes than bilateral smiling at electrode Cz. Similarly, left-sided finger movements led to greater negativity than right-sided finger movements at electrode $\mathrm{Cz}$. However, amplitudes at $\mathrm{Cz}$ for right finger and bilateral smiling movements did not differ from each other. These findings may be explained in terms of movement complexity, and are in concordance with previous reports of stronger NS' before movements, which participants felt to be more difficult to execute [15]. Speculatively, in addition to finding unilateral smiling more difficult than bilateral smiling, our participants (which were all right-handed) may have had fewer difficulties in performing repetitive movements with their right than with their left index finger. Moreover, differences for right and left finger movements may also be explained by the fact that right-handed subjects recruit both hemispheres for moving their left hand, while they activate only the left (pre)motor cortex when moving their right hand [28]. Of course, a way to test amplitude differences between the dominant and non-dominant hand would have been to compare the BP in right- and left-handed participants.

The predicted pattern of amplitude increase over the contralateral hemisphere for unilateral smiles reached only marginal significance, the difference between amplitudes at electrodes C3 and C4 was significantly different from 0 for right-sided, but not for left-sided smiles, and finally the topographical maps revealed a less pronounced contralateral shift for unilateral smiles than for unilateral finger movements (see Fig. 1b). Studies in nonhuman primates suggest that these effects may be due to the fact that voluntary movements of the lower facial muscles 
rely-besides on the primary motor cortex - partly also on medial motor structures like the caudal cingulate motor cortex (see [29]). These medial motor structures may be less involved in the performance of voluntary finger movements.

We extracted for each condition the map representing voltage fluctuations over all electrodes at time of EMGonset $( \pm 10 \mathrm{~ms})$, and confirmed its specificity for the corresponding condition - with exception of the map for right-sided smile. The fact that we could not confirm the specificity of the map for right-sided posed smiles may be attributable to the relative small number of trials per condition and participant. Nevertheless, the overall results of this experiment make us confident in the quality of our data. For example, the symmetrical distribution of scalpnegativity before bilateral smiling fits well with what is known about the cortical innervation of lower-face muscles, and is most likely due to a synchronous activation of the right and left motor cortices.

The ecological validity of our results may be questionable, as smiles may not often be prepared several seconds in advance, outside of the laboratory. This drawback, however, applies to most of the studies which used the Bereitschaftspotential-paradigm, and should not discourage us from pursuing laboratory experiments. Similarly, one could argue that unilateral smiles are quite rare in everyday life, and are not related to a genuine emotional expression. Indeed, many of our participants reported this kind of movements to be difficult to execute. However, our main interest here was on the BP concerning the bilateral, thus usual smile. Importantly, we only asked participants to produce posed (i.e. unemotional) smiles. This kind of posed facial expression may sometimes be rather unilateral in some persons and/or situations. Indeed, posed smiles have long been known to be more asymmetrical compared to truly felt smiles [30].

A further potential criticism to this study is that solely the activity of the zygomaticus muscles was assessed, and therefore co-contractions (especially during unilateral smiles, which were reportedly difficult to execute) of other (facial) muscles cannot be excluded. On the other hand, it seems unlikely that all participants co-activated other muscles in a systematic way, which would have affected the analyses. Still, future experiments of this kind should control for co activations of non-target muscles.

Finally, partly due to the small number of trials available, our analyses focused on amplitude and topography differences at time of movement onset (targeting the NS' and MP). Of further interest would be to compare for example onsets and amplitudes of the various pre-movement potentials (BP, NS', MP) across movement types. Moreover, the precise neural generators of these potentials and their eventual shift depending on the muscles activated could be investigated through inverse solution methods
[22]. As noted above, some lines of study suggest that a BP precedes not only voluntary but also spontaneous movements [9]. It would therefore be of interest to further explore the characteristics of premotor potentials across different types of movement, and especially across different types of emotional facial expressions.

In summary, for the first time, we have shown the neural correlates of the motor preparation and early execution of posed smiles. We identified a late BP which is comparable to the one preceding finger, hand, or other distal movements. The BP before posed smiles however also possesses specific characteristics, such as a later onset, lower amplitude (except for the comparison with right index movements) and a specific topography with a symmetrical bilateral distribution at time of movement onset.

Acknowledgements Many thanks to Lucas Tamarit for help with Analyses and Images. The Cartool software (http://brainmapping. unige.ch/Cartool.htm) has been programmed by Denis Brunet, from the Functional Brain Mapping Laboratory, Geneva, Switzerland, and is supported by the Center for Biomedical Imaging (CIBM) of Geneva and Lausanne.

\section{References}

1. Scherer KR. What are emotions? And how can they be measured? Soc Sci Inf 2005;44:695-729.

2. Ekman P, Rosenberg EL (editors). What the face reveals. New York: Oxford University Press; 2005.

3. Hopf HC, Muller-Forell W, Hopf NJ. Localization of emotional and volitional facial paresis. Neurology 1992;42:1918-23.

4. Rinn WE. The neuropsychology of facial expression: a review of the neurological and psychological mechanisms for producing facial expressions. Psychol Bull 1984;95:52-77.

5. Kornhuber HH, Deecke L. Hirnpotentialänderungen beim Menschen vor und nach Willkürbewegungen, dargestellt mit Magnetbandspeicherung und Rückwärtsanalyse. Pflugers Arch Gesamte Physiol Menschen Tiere 1964;281:52.

6. Castro A, Diaz F, van Boxtel GJ. What happens to the readiness potential when the movement is not executed? Neuroreport 2005;16:1609-13.

7. Jankelowitz SK, Colebatch JG. Movement-related potentials associated with self-paced, cued and imagined arm movements. Exp Brain Res 2002;147:98-107.

8. Galdo-Alvarez S, Carrillo-de-la-Pena MT. ERP evidence of MI activation without motor response execution. Neuroreport 2004;15:2067-70.

9. Keller I, Heckhausen H. Readiness potentials preceding spontaneous motor acts: voluntary vs. involuntary control. Electroencephalogr Clin Neurophysiol 1990;76:351-61.

10. Libet B, Wright EW Jr, Gleason CA. Readiness-potentials preceding unrestricted 'spontaneous' vs. pre-planned voluntary acts. Electroencephalogr Clin Neurophysiol 1982;54:322-35.

11. Shibasaki H, Hallett M. What is the Bereitschaftspotential? Clin Neurophysiol 2006;117:2341-56.

12. Shibasaki H, Barrett G, Halliday E, Halliday AM. Components of the movement-related cortical potential and their scalp topography. Electroencephalogr Clin Neurophysiol 1980;49:213-26.

13. Cui RQ, Deecke L. High resolution DC-EEG analysis of the Bereitschaftspotential and post movement onset potentials 
accompanying uni- or bilateral voluntary finger movements. Brain Topogr 1999;11:233-49.

14. Ikeda A, Luders HO, Shibasaki H, Collura TF, Burgess RC, Morris HH, Hamano T. Movement-related potentials associated with bilateral simultaneous and unilateral movements recorded from human supplementary motor area. Electroencephalogr Clin Neurophysiol 1995;95:323-34.

15. Kitamura J, Shibasaki H, Kondo T. A cortical slow potential is larger before an isolated movement of a single finger than simultaneous movement of two fingers. Electroencephalogr Clin Neurophysiol 1993;86:252-8.

16. Yamamoto J, Ikeda A, Satow T, Matsuhashi M, Baba K, Yamane F, Miyamoto S, Mihara T, Hori T, Taki W, Hashimoto N, Shibasaki H. Human eye fields in the frontal lobe as studied by epicortical recording of movement-related cortical potentials. Brain 2004;127:873-87.

17. Huckabee ML, Deecke L, Cannito MP, Gould HJ, Mayr W. Cortical control mechanisms in volitional swallowing: the Bereitschaftspotential. Brain Topogr 2003;16:3-17.

18. Nakajima I, Tanaka Y, Uchida A, Sakai T, Akasaka M, Mori A, Sumino R. Cortical potentials associated with voluntary biting movement in humans. Neurosci Res 1991;10:285-9.

19. Yoshida K, Kaji R, Hamano T, Kohara N, Kimura J, Shibasaki H, Iizuka T. Cortical potentials associated with voluntary mandibular movements. J Dent Res 2000;79:1514-8.

20. Wohlert AB. Event-related brain potentials preceding speech and nonspeech oral movements of varying complexity. J Speech Hear Res 1993;36:897-905.

21. Morecraft RJ, Louie JL, Herrick JL, Stilwell-Morecraft KS. Cortical innervation of the facial nucleus in the non-human primate: a new interpretation of the effects of stroke and related subtotal brain trauma on the muscles of facial expression. Brain 2001;124:176-208.

22. Michel CM, Murray MM, Lantz G, Gonzalez S, Spinelli L, Grave de Peralta R. EEG source imaging. Clin Neurophysiol 2004;115:2195-222.

23. Khateb A, Abutalebi J, Michel CM, Pegna AJ, Lee-Jahnke H, Annoni JM. Language selection in bilinguals: a spatio-temporal analysis of electric brain activity. Int J Psychophysiol 2007; 65:201-13.

24. van Boxtel A. Optimal signal bandwidth for the recording of surface EMG activity of facial, jaw, oral, and neck muscles. Psychophysiology 2001;38:22-34.

25. Gratton G, Coles MG, Donchin E. A new method for off-line removal of ocular artifact. Electroencephalogr Clin Neurophysiol 1983;55:468-84.

26. Deecke L, Grozinger B, Kornhuber HH. Voluntary finger movement in man: cerebral potentials and theory. Biol Cybern 1976;23:99-119.

27. Cui RQ, Huter D, Lang W, Deecke L. Neuroimage of voluntary movement: topography of the Bereitschaftspotential, a 64-channel DC current source density study. Neuroimage 1999;9:124-34.

28. Heilman KM, Meador KJ, Loring DW. Hemispheric asymmetries of limb-kinetic apraxia: a loss of deftness. Neurology 2000;55: $523-6$.

29. Morecraft RJ, Stilwell-Morecraft KS, Rossing WR. The motor cortex and facial expression: new insights from neuroscience. Neurologist 2004;10:235-49.

30. Ekman P, Friesen WV. Felt, false, and miserable smiles. J Nonverbal Behav 1982;6:238-58. 